\title{
ERRATA: MAL'CEV CONDITIONS, SPECTRA AND KRONECKER PRODUCT
}

\author{
WALTER D. NEUMANN
}

(Received 28 May 1979)

I am grateful to W. Taylor for pointing out that Lemma 2.3 of Neumann (1978), which claimed that a variety $\mathscr{V}$ and its finitary reduct $\mathscr{V}^{t}$ have the same finite algebras, is incorrect. This weakens several results of the paper: the lattice homomorphism of Lemma 3.1 is not complete, Theorem 4.1 is false, and the Mal'cev conditions claimed to be strong in 6.1, 6.2 and 7.1 are not strong. Taylor (1980) shows one cannot improve on this.

W. Taylor and A. Bateson also point out that the proof of 5.4 is incomplete, since the cell structure on the topological algebra $F(X)$ described there does not carry the weak topology, so the standard presentation of fundamental group may not apply. Though this gap can probably be filled, the technical difficulties in a correct proof by Bateson (1977) of a somewhat weaker version of this theorem indicate that it may be quite hard to do so.

\section{References}

A. Bateson (1977), 'Interplay of algebra and topology', Thesis, University of Colorado.

W. D. Neumann (1978), 'Mal'cev conditions, spectra and Kronecker product', J. Austral. Math. Soc. Ser. A 25, 103-117.

W. Taylor (1980), 'Mal'tsev conditions and spectra', J. Austral. Math. Soc. Ser. A. (to appear).

Department of Mathematics

University of Maryland

Maryland 20742

U.S.A. 